\title{
Predictive Control Algorithm Based on Integral Action- Design and Implementation on a Conical Tank System
}

\author{
Vinayambika S Bhat ${ }^{1}$, I. Thirunavukkarasu ${ }^{1}$, S. Shanmuga Priya ${ }^{2}$ and Shreesha $C^{1}$ \\ ${ }^{1}$ Dept. of Instrumentation and Control Engineering, Manipal Institute of Technology, Manipal University, Manipal- 576104, Karnataka, India \\ ${ }^{2}$ Dept. of Chemical Engineering, Manipal Institute of Technology, Manipal University, Manipal- 576104, Karnataka, India
}

\begin{abstract}
This article presents a Model Predictive Control (MPC) algorithm based on integral action. Level control in process industry is challenging because of nonlinearity presents in the shape of the tank, actuators etc. The conical tank system is taken as benchmark process in the present study. It is Single Input Single Output (SISO) nonlinear system whose cross-sectional area varies along the tank height. The control algorithm is simulated using MATLAB $\mathrm{m}$-file environment. The effectiveness of the predictive algorithm is also presented by experimentally validating it on a conical tank system at different heights. The interfacing of $\mathrm{m}$-file with the experimental setup is the challenging task faced during the initial stage of experimental validation.
\end{abstract}

\section{Introduction}

A basic problem in process industries is control of liquid level and flow in process tank. Conical tanks are extensively used in industries like petrochemical, food, wastewater treatment and hydrometallurgical industries [1]. Also, control of conical tank is a challenging problem because of its nonlinearity and constantly changing area of cross-section [2, 3]. Advanced and innovative algorithms are required for process control applications. Literature discusses that predictive control algorithms are effectively used in process industries $[4,5]$. There are several methods within MPC algorithms that differ from each other based on the process model used [6]. However, these algorithms have some problems with offset i.e. the process output is not equal to the specified reference at steady state [7]. This offset problem can be solved by introducing the integral action [8]. The MPC with integral action algorithm is designed and implemented for conical tank system. The state space model of the conical tank system is considered for the study. The predictive algorithm is simulated in MATLAB environment. Also, the effectiveness of MPC with integral action has been validated by implementing it in real-time.

The article is outlined as follows. A brief introduction to predictive control algorithm is given section II. Section III presents details of the experimental setup. The methodology involved in developing the MPC integral action algorithm is discussed in section IV. Both the simulation and real-time results are presented and discussed in section $\mathrm{V}$ followed by conclusion in section VI. Also, in the following section, scope for future work in the reported research area is noted.

\section{Fundamentals of predictive control algorithm}

The basic structure of predictive control algorithm is shown in Figure 1. The main motive of predictive control algorithm is to find the input signal that best satisfies to a given performance criterion (cost function), it predicts how the system will behave if the signal is applied. It solves a control problem for an optimal control action to track the given input [5]. Models are used to predict the behaviour of dependent variables or outputs of the modelled dynamical system with respect to changes in the process independent variables or inputs. The main distinguishing factor of these predictive algorithms is their ability to deal with explicit constraints on system variables that are neglected in majority of the other control algorithms [7].

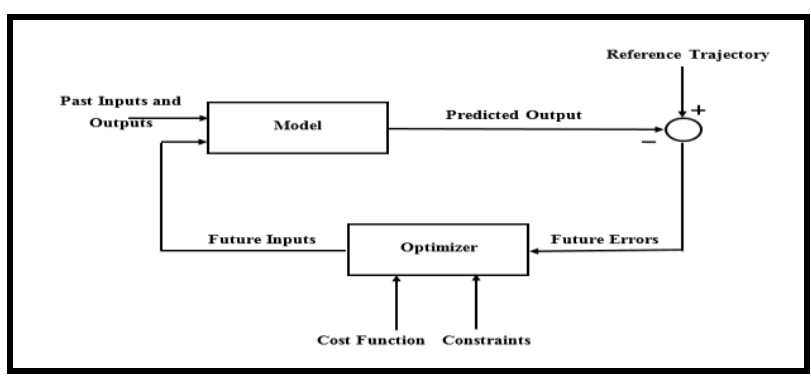

Figure 1. Basic structure of predictive control algorithm [5]

\section{Experimental setup}


The Conical Tank Level control system chosen for the study is shown in Figure 2. The Figure 2(a) represents the structure of conical tank followed by the experimental setup in Figure 2(b). The setup consists of level transmitter, pump, rotameter, conical tank, reservoir and power supply. The system descriptions are given in Table 1. The conical shape of the tank makes the level process nonlinear, since the area is directly proportional to the level. The tank is modelled by dividing the total height into four regions with $7 \mathrm{~cm}$ for each region. The linearized model for the regions between $7 \mathrm{~cm}-14 \mathrm{~cm}$ is obtained using the 2-point method. The VDAS-01 interfacing card is used for the real- time implementation of predictive control algorithm. The Data Acquisition Card (DAC) is used to interface the $\mathrm{PC}$ with the real-time system is shown in Figure 3. The MATLAB m-file code (Control algorithm developed) has been interfaced through conical tank system for the real-time implementation. The controller output is limited to 0-250. The First Order Plus Time Delay (FOPTD) model of the conical tank system for the second region $(7-14 \mathrm{cms})$ of locally lineralized model is given by Eq. (1) [2].

$G(s)=\frac{0.925 e^{-1.09 s}}{25.05 s+1}$

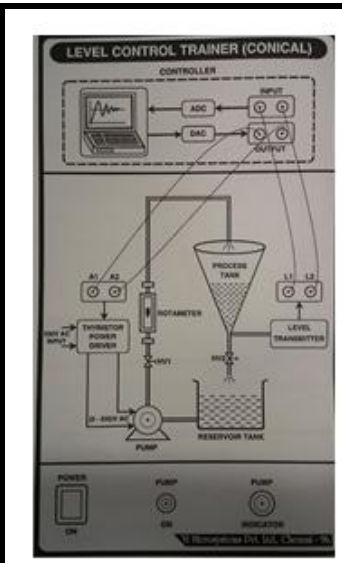

(a)

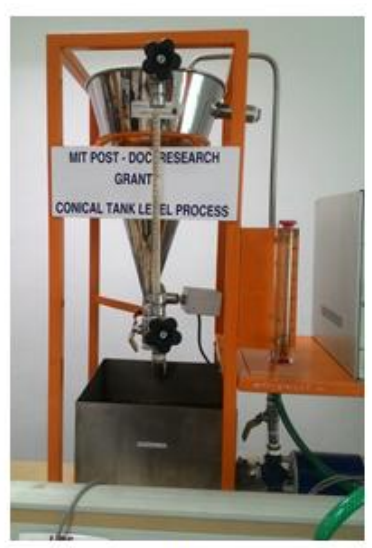

(b)
Figure 2. (a) Structure of the conical tank (b) Experimental setup of conical tank [2]

Table 1. Technical specification of conical tank setup [2]

\begin{tabular}{|c|c|}
\hline Conical Tank & Motor \\
\hline Height- 33cms & RPM 1500 \\
\hline Top Diameter-30cms & Head 24M \\
\hline Bottom Diameter- 3cms & LPH 1600 \\
\hline
\end{tabular}

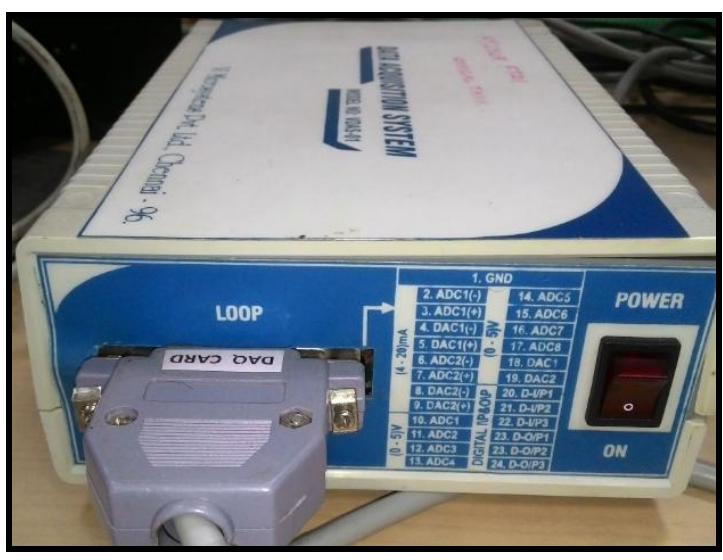

Figure 3. VDAS-01 Data acquisition card used for the realtime implementation

\section{Methodology}

A state space representation of the system has been obtained from the transfer function of the system. The process model can be described as follows.

$$
\begin{aligned}
& x_{k+1}=A x_{k}+B u_{k}+v \\
& y_{k}=C x_{k}+w
\end{aligned}
$$

Where A, B and C are the state space representation of the system of appropriate dimensions. The $\mathrm{v}$ and $\mathrm{w}$ are the disturbances which are unknown. That means, $\mathrm{v}$ may be an unknown constant or slowly varying process disturbance, and $\mathrm{w}$ may be an unknown constant or slowly varying noise vector. Variables $y_{k}$ and $u_{k}$ in the model Eq. (2) are the actual input and output variables respectively. To solve the MPC optimal control problem for Eq. (2) a model which is independent of the unknown disturbances has been considered [8]. That is Eq. (2) has been reframed as

$\Delta x_{k+1}=A \Delta x_{k}+B \Delta u_{k}$

$y_{k}=y_{k-1}+C \Delta x_{k}$

Where, $\Delta x_{k}=x_{k}-x_{k-1}$

Augmenting Eqs. (3) with (4) gives the state space model:

$$
\begin{aligned}
& {\left[\begin{array}{c}
\Delta x_{k+1} \\
y_{k}
\end{array}\right]=\left[\begin{array}{ll}
A & 0_{n \times m} \\
C & I_{m \times m}
\end{array}\right]\left[\begin{array}{c}
\Delta x_{k} \\
y_{k-1}
\end{array}\right]+\left[\begin{array}{c}
B \\
0_{m \times r}
\end{array}\right] \Delta u_{k}} \\
& y_{k}=\left[\begin{array}{lll}
C & I_{m \times m}
\end{array}\right]\left[\begin{array}{l}
\Delta x_{k} \\
y_{k-1}
\end{array}\right]
\end{aligned}
$$


The final state space model is as follows.

$$
\Delta \bar{x}_{k+1}=\bar{A} \Delta \bar{x}_{k}+\bar{B} \Delta u_{k}
$$

$y_{k}=C \Delta \bar{x}_{k}$

The state space model Eqs. (7) and (8) is used to define a Prediction Model (PM) of the form given by

$$
y_{k+1 / L}=p_{L}+F_{L} \Delta u_{k / L}
$$

Where, $p_{L}=O_{L} \bar{A} \bar{x}_{k}, F_{L}=\left[\begin{array}{ll}O_{L} \bar{B} & H_{L}^{d}\end{array}\right]$, OL is the extended observability matrix of the pair $\bar{A}, \bar{C}$ and $H_{L}^{d}$ is the Toeplitz matrix. The cost function along with control law is expressed as follows.

$$
J=\Delta u_{k / L}^{T} H \Delta u_{k / L}+2 f_{k}^{T} \Delta_{k / L}
$$

Where, $H=F_{L}^{T} Q F_{L}+P$ and $f_{k}=F_{L}^{T} Q\left(p_{L}-r_{k+1 / L}\right)$. The $\mathrm{P}$ and $\mathrm{Q}$ are the weighting function selected by trial and error to achieve the stable closed loop response.

The unconstrained MPC control is given by

$$
\Delta u_{k / L}^{*}=-H^{-1} f_{k}
$$

The control law is given by

$$
u_{k / L}=S \Delta u_{k / L}+c u_{k-1}
$$

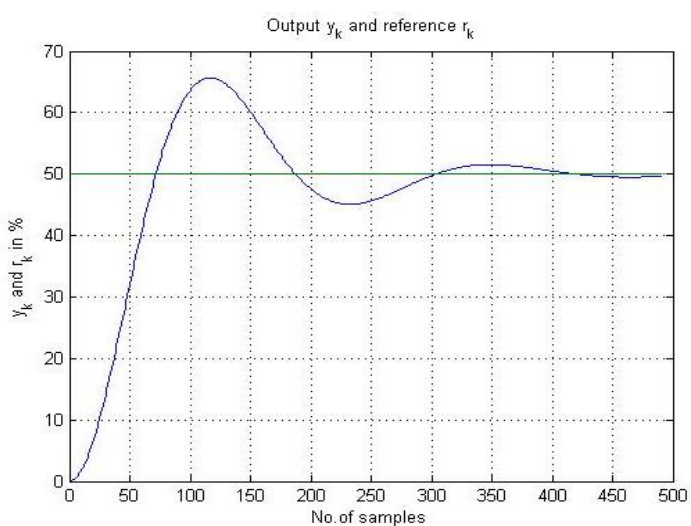

(a)
Here, $S \in R^{r L \times r L}$ and $c \in R^{r L \times r}$ are the matrices with ones and zeros. The MPC algorithm minimizes the cost function with respect to the constraints. This is a standard Quadratic Programming (QP) problem in terms of unknown future control increments and optimal solution is $\Delta u_{k / L}^{*}$ as defined in Eq. (11). A receding horizon strategy is used and only the first control increment $\Delta u_{k}^{*}$ in the calculated $\Delta u_{k / L}^{*}$, is used for control. The actual control action to the process is then $u_{k}=\Delta u_{k}^{*}+u_{k-1}$.The structure of linear quadratic controller is proportional to the integral form as in case of conventional PI control algorithm $[9,10]$. Thus, the name predictive control algorithm with integral action [8].

\section{Results and discussion}

A state space equivalent model has been considered to develop the control algorithm in simulation. The corresponding A, B, C, D matrix in discrete domain for sampling period of 0.5 is given by

$\mathrm{A}=[0.9802], \mathrm{B}=[0.125], \mathrm{C}=[0.1439], \mathrm{D}=[0.01502]$

The MPC with integral action algorithm has been successfully implemented on the real-time system of SISO Conical Tank Level system and the output could track the reference signal. The output prediction and corresponding control input for setpoint of $50 \%$ is shown in Figure 4. Also, for the setpoint 70, the predicted output and the corresponding control input is shown in Figure 5. The plant output and manipulated variable response for setpoints $50 \%$ and $70 \%$ of tank level are shown in Figures 6 and 7 respectively.

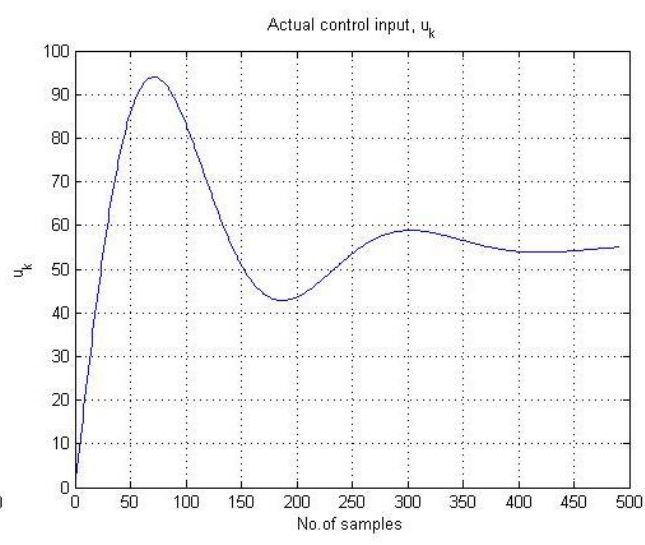

(b)

Figure 4. (a) Output prediction vs number of samples at setpoint of 50 (b) Actual control input $u_{k}$ vs number of samples 


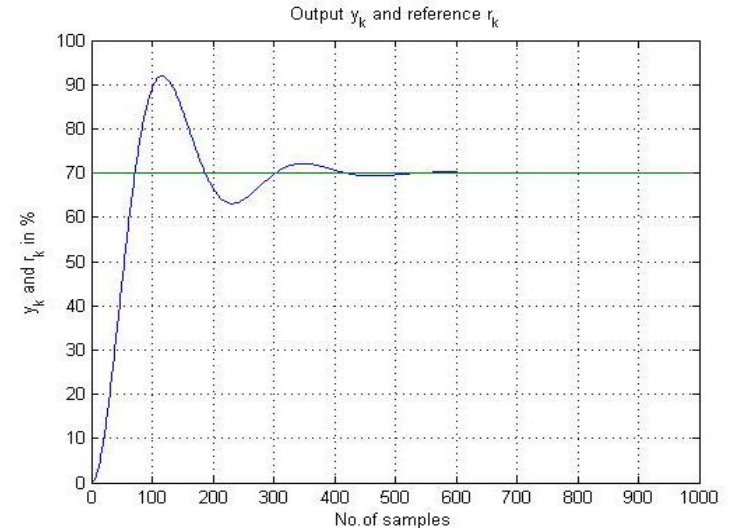

(a)

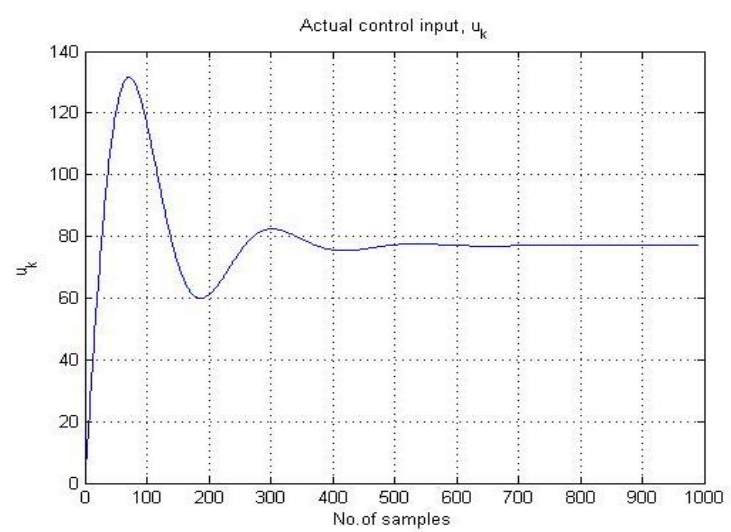

(b)

Figure 5. (a) Output prediction vs number of samples at setpoint of 70 (b) Actual control input $u_{k}$ vs number of samples

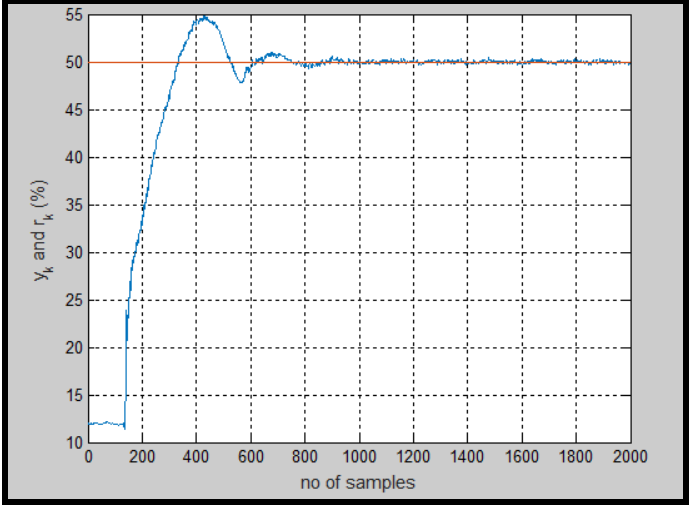

(a)

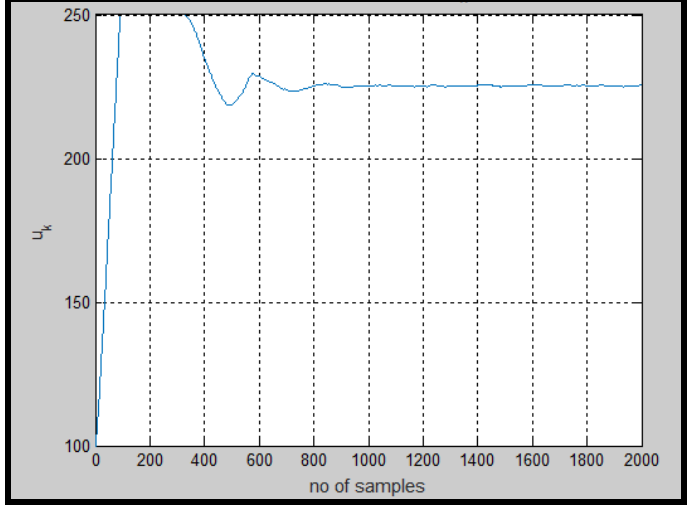

(b)

Figure 6. (a) Experimental Process output and (b) Manipulated variable response for a setpoint of 50\%

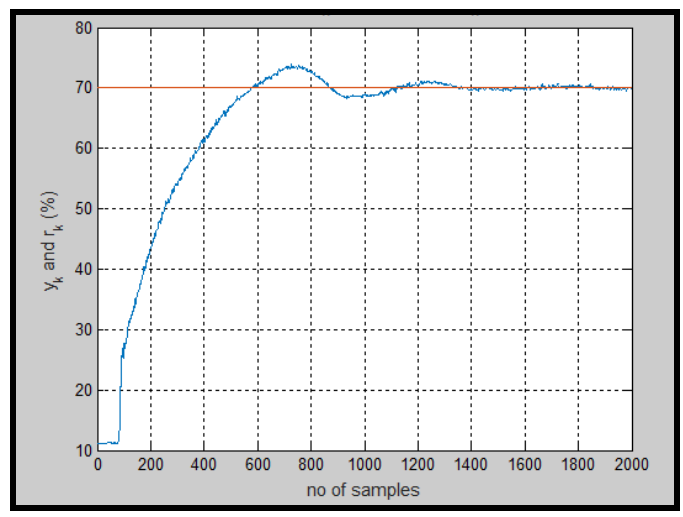

(a)

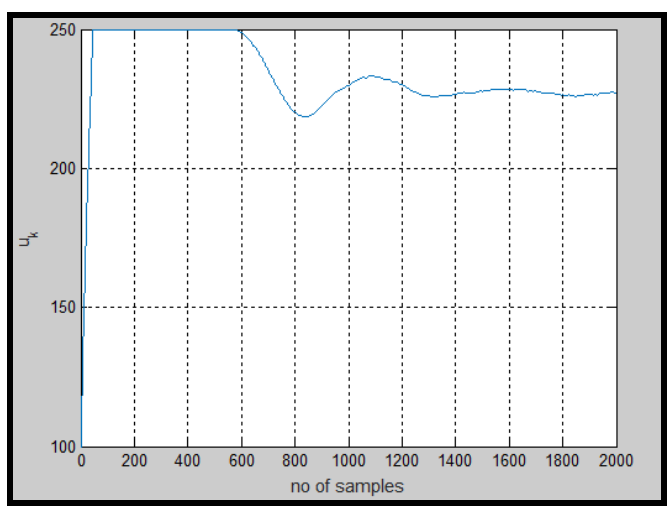

(b)

Figure 7. (a) Experimental Process output and (b) Manipulated variable response for a setpoint of 70\%

\section{Conclusion}

The predictive control algorithm along with integral action is considered for the design and experimental validation. The state space model of the system is obtained from the transfer function of the system. The effectiveness and practicality of the control algorithm is studied and simulated by using conical tank plant model.
The closed loop servo response is obtained in simulation. The MATLAB m-file predictive control algorithm is interfaced to real-time using data acquisition systems VDAS-01. The same setpoint is maintained both in simulation and real-time for the comparison. The challenges faced during the m-file interface to the DAQ card and experimentation will be addressed during the presentation. 


\section{Future work}

The MPC with integral action is extended to MIMO system. The control algorithm simulated and validated on a pilot plant binary distillation column. It was reported by the authors in the research article submitted to CHEMCON-2017, HIT, Haldia (Accepted for oral presentation) with experimental results of pilot plant binary distillation column. Few issues have been observed as drawbacks to retune the controller.

\section{Acknowledgement}

Vinayambika S. Bhat would like to acknowledge the Mangalore Institute of Technology and Engineering (MITE), Moodabidri, for sponsoring her Ph.D. programme. The authors would like to thank Department of Instrumentation and Control Engineering, MIT, Manipal University for providing the real-time experimental facility for carrying out the experimental work.

\section{References}

1. C. Lovaas, M. M. Seron, and G. C. Goodwin, Robust Output-Feedback MPC with Integral Action, IEEE Transactions on Automatic Control, 55(7), pp. 15311543, (2010).

2. M. K. Rakesh, R. S. Babu and I. Thirunavukkarasu, Sliding Model with Dead Time Compensation for Conical Tank Level Process, IOSR Journal of Electrical and Electronics Engineering, 9(2), pp. 5964 (2014).
3. V. R. Ravi, T. Thyagarajan and G. Uma Maheshwaran, Dynamic Matrix Control of a Two Conical Tank Interacting Level System, Proceedia Engineering, 38, pp. 2601-2610 (2012).

4. N. Sivakumaran and T. K. Radhakrishnan, Predictive Controller Design for Non-Linear Chemical Processes, Indian Journal of Chemical Engineering, 14, pp. 341-349 (2007).

5. V. S. Bhat, I. Thirunavukkarasu, and S. Shanmuga Priya, Design of Unconstrained DMC to Improve the Distillate Product Purity of the Distillation Column, MATEC Web of Conferences, 77, pp. 1-5, presented in ICPEME, Thailand, June (2016).

6. M. Manimaran., S. Malaisamy, M. M. Rafiq, V. Petchithai, V. S. Chitra, K. Kalanithi. and H. Abirami., Parameter Identification and Dynamic Matrix Control Design for a Nonlinear Pilot Distillation Column, International Journal of ChemTech Research, 7(1), pp. 382-388 (2015).

7. P. E. Orukpe, Model Predictive Control Fundamentals, Nigerian Journal of Technology, 31(2), pp. 139-148, (2012).

8. D. D. Ruscio, Model Predictive Control with Integral Action: A Simple MPC Algorithm, Modeling, Identification and Control, 34(3), pp. 119-129, (2013).

9. D. D. Ruscio, Discrete LQ Optimal Control with Integral Action: A Simple Controller on Incremental form for MIMO Systems, Modeling, Identification and Control, 33(2), pp. 35-44, (2012).

10. D. D. Ruscio, Model Predictive Control and Identification: A Linear State Space Model Approach, in Proceedings of the $36^{\text {th }}$ conference on Decision and Control, USA, 4, pp. 3202-3209, December (1997). 